\title{
Situación legislativa de la Violencia obstétrica en América latina: el CASO de Venezuela, Argentina, MéXico y Chile
}

[Legislative situation of Obstetric violence in Latin America: the case of Argentina, Venezuela, México and Chile]

\author{
Luis IVÁn Díaz García* \\ Universidad Católica de Temuco \\ Yasna Fernández M.**
}

\begin{abstract}
RESUMEN
El artículo revisa la situación legislativa de la violencia obstétrica en América Latina. En particular analiza aquellos países que cuentan con legislación específicamente destinada a enfrentarla (Venezuela, Argentina y México). El análisis incluye las normas chilenas que pueden tener relación con este tema.

\section{Palabras clave}

Violencia obstétrica - violencia de género - derechos fundamentales - Derecho comparado - Derecho sanitario. Abstract
\end{abstract}

The article examine the obstetric violence legislative situation in Latin America. Particularly analyzes those countries that have a legislation specifically aimed to deal with it (Venezuela, Argentina and México). The analysis includes Chilean rules that could have relation whit this issue.

\section{KEY WORDS}

Obstetric violence - gender violence - fundamental Rights - Comparative Law - Health Law.

Recibido el 25 de octubre de 2018 y Aprobado el 8 de noviembre de 2018

* Académico, Facultad de Ciencias Jurídicas, Universidad Católica de Temuco. Doctor en Derecho, Universidad Carlos III de Madrid, España. Abogado, Pontificia Universidad Católica de Chile. Dirección postal: Montt 56, Temuco. Correo electrónico: ivandiaz@uct.cl.

** Abogada, Licenciada en Ciencias Jurídicas y Magíster en Derecho, Universidad Autónoma de Chile. Diplomado en Problemas actuales del Derecho Penal y Procesal Penal, Universidad Católica de Temuco. Diplomado en Reconocimiento y Protección Jurídica de los Derechos de la Infancia y Adolescencia Indígena. UNICEF. - Universidad Católica de Temuco. Dirección postal: Montt 56, Temuco. Correo electrónico: yasnaf@hotmail.com. 


\section{INTRODUCCIÓN}

\section{Objetivo, pregunta e hipótesis}

La violencia obstétrica es una manifestación de mala práctica más o menos reciente en el ámbito sanitario, relacionado con el traslado del parto desde el hogar a los establecimientos de salud ${ }^{1}$. Por lo mismo, no es de extrañar que no haya sido objeto de mayor discusión en las revistas científicas de Derecho o de Medicina en Chile 2 . Y todo ello, pese a la gravedad de este silencioso atentado contra la dignidad y derechos fundamentales de las mujeres.

En ese contexto, el objetivo del presente artículo es analizar la situación normativa de la violencia obstétrica en América Latina, singularmente en aquellos que han establecido regulaciones que pretenden enfrentar directamente esta forma de agresión contra la mujer. La revisión se extiende a la situación chilena, aun cuando no cuente con una tal regulación.

En línea con dicho objetivo, la pregunta que orienta la investigación puede ser formulada de la siguiente manera: ¿Cuál es la situación normativa y fáctica de la violencia obstétrica en los países de América Latina que han legislado sobre la materia, y particularmente en Chile?

La hipótesis de trabajo es la siguiente: en América Latina muy pocos países han establecido regulaciones que pretendan enfrentar directamente el fenómeno de la violencia obstétrica y Venezuela, México y Argentina, que lo han hecho, han alcanzado un escaso impacto. Chile, por su parte, carece de una tal regulación y existen importantes indicios de un elevado grado de prevalencia del problema.

\section{Metodología}

Para responder la pregunta que guía la presente investigación y determinar si se confirma la hipótesis en las conclusiones, se ha seguido la siguiente metodología.

En primer lugar se estableció un marco conceptual que permita precisar en qué consiste la violencia obstétrica a partir de su significado y las formas en que se manifiesta. Al explicitar estos aspectos se especifica de qué se está hablando cuando se utiliza aquella expresión.

1 Camacaro, M., Patologizando lo natural, naturalizando lo patológico... improntas de la praxis obstétrica, en Revista venezolana de estudios de la mujer 14 (2009), 32, p. 149.

${ }^{2}$ La revisión de las revistas chilenas de Derecho y de Ciencias de la Salud indexadas en SciELO evidencia que no existen artículos sobre la materia al menos hasta el primer semestre de 2016. En el buscador de cada una de ellas se insertó la expresión "violencia obstétrica" sin obtener resultados. 
En segundo lugar se revisaron las páginas web oficiales de los poderes legislativos de todos los países de América Latina, para determinar en cuál de ellos existían regulaciones relativas a la violencia obstétrica. Esta exploración permitió confirmar que al año 2016 sólo tres de esos países cuenta con legislación sobre la materia: Venezuela, Argentina y México. En este último país las normativas son emitidas a nivel de los Estados que integran la Unión y no a nivel Federal. En ellos se centra la presente investigación. La inclusión de Chile, pese a carecer de una regulación específicamente dirigida a enfrentar la violencia obstétrica, se justifica en la sede en que se pretende publicar esta investigación.

En tercer lugar se realizó una búsqueda bibliográfica relativa a la regulación existente en los tres países mencionados y en chile, y a la situación fáctica de la violencia obstétrica en los mismos. Para este efecto se revisaron todas las publicaciones sobre la materia indexadas en $\mathrm{SciELO}^{3}$ y las demás no indexadas disponibles en Internet hasta junio de 2016, que proveyeran información sobre el tópico. Al efecto se insertaron en el buscador "Google" las expresiones "violencia obstétrica scielo", agregándose luego y de manera sucesiva, para depurar la búsqueda, las expresiones "Venezuela", "México", "Argentina" y "Chile". Esto permitió ubicar 9 artículos publicados en SciELO y 8 artículos publicados en otras fuentes que resultaban útiles para el estudio.

Con miras a delimitar los alcances de esta investigación, conviene decir todavía dos cosas más. En primer lugar se debe reiterar que hasta la fecha de cierre de esta investigación (2016) la realidad normativa evidencia que la violencia obstétrica carece de regulación en la mayor parte de los países de América Latina. Es por ello que la exposición se limita a la legislación de los tres países ya mencionados (Venezuela, Argentina y México), a los que se agrega Chile. En segundo lugar se debe reconocer que la violencia obstétrica también puede recaer sobre el niño que está por nacer o recién nacido. Sin embargo, esta forma de violencia, tan o más grave que la dirigida contra la mujer, no será objeto de análisis en este momento.

\section{Plan de la investigación}

En línea con lo que se viene expresando, la exposición se vertebra en torno a tres apartados. En el primero de ellos se revisan los aspectos esenciales de la violencia obstétrica (apartado II). El segundo expone la situación normativa de los tres países Latinoamericanos que han pretendido

${ }^{3}$ SciELO es la abreviatura de Scientific Electronic Library Online. Se trata de un buscador de libre acceso on line, gratuito, al que se adscriben revistas de diversos países Iberoamericanos. 
enfrentar la violencia obstétrica y, hasta donde es posible, su resultado, como así también la situación normativa en Chile (apartado III). El último ofrece algunas conclusiones que, asentadas en la investigación realizada, permiten confirmar la hipótesis sostenida (apartado IV).

\section{ASPECTOS ESENCIALES DE LA VIOLENCIA OBSTÉTRICA}

\section{Concepto de violencia obstétrica}

La violencia obstétrica es una forma de violencia contra la mujer, de modo que el género ha sido esencial en su conceptualización ${ }^{4}$. La violencia contra la mujer es aquella agresión que se dirige en contra de ella precisamente por pertenecer al género femenino 5 . La violencia de género normalmente es ejercida por un varón, pero también puede ser ejercida por una mujer, pues lo relevante es que la víctima se encuentra en una situación de desventaja en las relaciones de poder. En el Derecho Internacional se ha declarado que "la violencia contra la mujer constituye una violación de los derechos humanos"6.

Siguiendo a Arguedas, la violencia obstétrica puede ser definida como "un conjunto de prácticas que degrada, intimida y oprime a las mujeres y a las niñas en el ámbito de la atención en salud reproductiva y, de manera mucho más intensa, en el período del embarazo, parto y postparto"7. El Observatorio de Equidad de Género en salud en Chile, por su parte, conceptualiza la violencia obstétrica como "aquella que ejerce el personal de salud sobre el cuerpo y los procesos reproductivos de las mujeres, expresada en un trato deshumanizado, un abuso de medicalización y patologización de los procesos naturales" ${ }^{\prime}$. Michelle Sadler y otros, yendo más allá, han

${ }^{4}$ Sadler, Michelle - Santos, Mário J.D.S. - Ruiz-Berdún, Dolores - Leiva Rojas, Gonzalo - Sкоко, Elena - Gillén, Patricia - Clausen, Jette A., Moving beyond disrespect and abuse: addressign the structural dimension of obstetric violence, en Reproductive Health Matters 24 (2016), p. 49.

${ }^{5}$ La Declaración sobre la Eliminación de la Violencia contra la Mujer, adoptada por la Asamblea de Naciones Unidas en 1993, inicia su artículo 1 de la siguiente manera: "A los efectos de la presente Declaración, por "violencia contra la mujer" se entiende todo acto de violencia basado en la pertenencia al sexo femenino".

${ }^{6}$ Preámbulo de la Convención Interamericana para Prevenir, Sancionar y Erradicar la violencia contra la mujer, de 1994.

7 Arguedas Ramírez, Gabriela, La violencia obstétrica: propuesta conceptual a partir de la experiencia costarricense, en Cuadernos Inter.c.a.mbio sobre Centroamérica y el Caribe 11 (2014), 1, p. 147.

8 Observatorio de Equidad de Género en Salud, Violencia de Género en Chile, Informe monográfico 2007-2012 ( ${ }^{\circ}$ 4, Santiago de Chile, OPS/OMS, 2013), p. 65. 
sostenido que la violencia obstétrica tiene el potencial de direccionar las dimensiones estructurales de la violencia, incluyendo las múltiples formas de insolencias y abusos durante el nacimiento?.

A partir de los conceptos recién transcritos resulta posible apreciar que las víctimas de la violencia obstétrica son las mujeres, sean niñas o adultas. Los sujetos activos de la violencia obstétrica, es decir, quienes la ejercen, son los profesionales del ámbito sanitario: médicos, matronas, enfermeros, otros profesionales de la salud y los estudiantes de esas mismas profesiones. El contexto en el que se produce esta forma de violencia de género es la atención profesional en el ámbito reproductivo y especialmente en situaciones de embarazo, parto y postparto. Aunque las razones de este fenómeno son diversas, especial mención merece la situación de saber-poder en que se encuentran los profesionales de la salud y frente a los cuales la mujer percibe que se debe limitar a obedecer. Las conductas son disímiles, y para una mejor precisión de las mismas conviene atender a las formas que puede adoptar la violencia obstétrica.

\section{Las formas de la violencia obstétrica}

La violencia obstétrica puede adoptar diversas formas. La que resulta más relevante a los fines del presente trabajo es aquella que distingue entre la física y la síquica (sin perjuicio que en algunos casos existe íntima conexión entre ambas), dividiéndose esta última en por acción y por omisión.

La violencia física es aquella que se ejerce sobre el cuerpo de la mujer. Entre las modalidades más habituales de esta forma de violencia se encuentran las siguientes: realizar prácticas invasivas o suministrar medicación que no esté justificada por el estado de salud de la persona que está por nacer o de la gestante, o sin autorización de esta última, no respetar los tiempos ni las posibilidades del parto biológico y, en íntima vinculación con ello, realizar la cesárea sin justificación estrictamente médica, proceder al tacto vaginal por más de una persona, utilizar fórceps sin estricta necesidad o consentimiento y realizar raspaje de útero sin anestesia ${ }^{10}$.

En cuanto a las cesáreas, conviene recordar que la Organización Mundial de la Salud ha recomendado que no excedan del 15\% del total de partos atendidos en un país ${ }^{11}$. La recomendación se funda en que: "tanto

9 Sadler, Michelle - Santos, Mário J.D.S. - Ruiz-Berdún, Dolores - Leiva Rojas, Gonzalo - Skoko, Elena - Gillén, Patricia - Clausen, Jette A., cit. (n. 4), p. 49.

${ }^{10}$ Medina, Graciela, Violencia obstétrica, en Revista de Derecho de Familia y de las Personas (2009), 2, p. 4; y BeLLI, Laura, La violencia obstétrica: otra forma de violación a los derechos humanos, en Redbioética 4 (2013), 1, p. 7.

${ }^{11}$ Organización Mundial de la Salud, Declaración de la OMS sobre tasas de 
las cesáreas como los partos instrumentales ponen en peligro la salud de la madre, del niño, o de ambos, y dejan secuelas físicas o psicológicas perdurables, de distinta gravedad, que pueden afectar también al establecimiento del vínculo madre-hijo, las relaciones sexuales de la mujer y hasta sus relaciones familiares" 12 .

La violencia psíquica es aquella que se ejerce sobre la estabilidad emocional de la mujer. Se produce tanto por acción como por omisión. Se produce por acción, por ejemplo, cuando la mujer es objeto de críticas por manifestar emociones como alegría o dolor, cuando es víctima de burlas sobre su estado, su cuerpo o su hijo, y, sobre todo, cuando se le impide plantear temores e inquietudes durante el proceso de embarazo y parto ${ }^{13}$. También hay violencia por acción cuando la víctima es objeto de un trato deshumanizado, grosero o humillante al pedir asesoramiento, al requerir atención o en el desarrollo de una práctica obstétrica.

La violencia psíquica se produce por omisión, por ejemplo, cuando a la mujer no se le informa sobre la evolución de su parto, sobre el estado de su hijo o hija o sobre las diferentes actuaciones de los profesionales ${ }^{14}$. También se produce la violencia psíquica por omisión, que es al mismo tiempo violencia física, si sobre la mujer se desarrollan prácticas quirúrgicas sin consentimiento previo y sin informar de sus ventajas y desventajas ${ }^{15}$, o mediando consentimientos que son obtenidos bajo circunstancias en las que la paciente no se encuentra en posición de rechazar ${ }^{16}$. De este modo la mujer se transforma en espectadora de lo que sucede con su propio cuerpo, debiendo aceptar y obedecer las decisiones ya tomadas.

cesárea de 1985, p. 2.

12 Fernández Guillén, Francisca, Nosotras parimos, ¿nosotras decidimos? El consentimiento de la mujer y otros aspectos legales de la atención materno infantil, en Medicina Naturista 10 (2006), p. 509.

13 Terán, Pablo - Castellanos, Carlos - González Blanco, Mireya - RAMos, Damarys, Violencia obstétrica: percepción de las usuarias, en Revista de Obstetricia y Ginecología de Venezuela 73 (2013), 3, p. 177.

${ }^{14}$ Medina, Graciela, cit. (n. 10), p. 4.

15 "A las pacientes se les realizan prácticas en muchos casos sin previa consulta, en otras ocasiones sin que se le brinde información sobre el estado de su salud, ni sobre las características de las intervenciones que se le realizarán. En consecuencia se le niega toda posibilidad de tomar decisiones alternativas, en algo tan íntimo y personal como es su salud, sexual y reproductiva”. Véase Medina, Graciela, cit. (n. 10), p. 1.

16 "Por otro lado, resulta ridículo pensar que los derechos de una mujer han quedado salvaguardados por el hecho de que se le pida que firme una hoja de consentimiento para practicar una cesárea cuando la necesidad de hacerla es consecuencia directa de prácticas sobre las que no ha tenido ninguna oportunidad de decidir". Fernández Guillén, Francisca, cit. (n. 12), p. 509. 


\section{Situación legislativa de la Violencia obstétrica en Venezuela, Argentina y MéXICO}

Según se indicó más arriba, sólo tres Estados cuentan con legislaciones específicamente dirigidas a enfrentar la violencia obstétrica: Venezuela, Argentina y México. A continuación se presentan los elementos que estructuran las legislaciones dictadas en cada uno de esos países y, hasta donde es posible en virtud de los escasos estudios disponibles, los efectos que han tenido en la práctica del personal de salud hacia las mujeres en contextos perinatales. Además, y según se anticipó, la misma revisión se hace respecto de Chile.

\section{Venezuela}

a) Regulación de la violencia obstétrica en Venezuela.- El 19 de marzo de 2007 se publicó en la Gaceta Oficial de Venezuela la Ley Orgánica sobre el Derecho de las mujeres a una vida libre de violencia ${ }^{17}$. Esta ley reconoce diecinueve formas de violencia contra la mujer, considerándose por entonces que la violencia obstétrica era la de regulación más novedosa ${ }^{18}$. En cuanto a dicha forma de violencia, se tratan esencialmente tres cosas: el concepto, las conductas constitutivas de violencia obstétrica y su sanción.

En cuanto al concepto, la ley expresa lo siguiente: "Se entiende por violencia obstétrica la apropiación del cuerpo y procesos reproductivos de las mujeres por personal de salud, que se expresa en un trato deshumanizador, en un abuso de medicalización y patologización de los procesos naturales, trayendo consigo pérdida de autonomía y capacidad de decidir libremente sobre sus cuerpos y sexualidad, impactando negativamente en la calidad de vida de las mujeres" $"$.

Las conductas constitutivas de violencia obstétrica contempladas en la regulación Venezolana son las siguientes: no atender oportuna y eficazmente las emergencias obstétricas, obligar innecesariamente a parir en posición supina y con las piernas levantadas, impedir sin causa el apego, alterar injustificadamente el proceso natural del parto mediante técnicas de aceleración y practicar cesáreas innecesarias. Además, y aunque erró-

${ }^{17}$ La Ley Orgánica sobre el Derecho de las mujeres a una vida libre de violencia sustituyó a la Ley sobre la Violencia contra la mujer y la familia de 1998.

18 Benítez Guerra, Gidder, Violencia obstétrica, en Revista de la Facultad de Medicina de la Universidad Central de Venezuela 31 (2008), 1, p. 5.

${ }_{19}$ Artículo 15, número 13, de la Ley Orgánica sobre el Derecho de las mujeres a una vida libre de violencia, de Venezuela. 
neamente no se le considera propiamente violencia obstétrica, se agrega la esterilización forzada ${ }^{20}$.

Por último, la ley regula dos clases de sanciones. Para la esterilización forzada se fija una pena privativa de libertad. Para las demás formas de violencia obstétrica se establecen penas pecuniarias ${ }^{21}$.

b) Situación fáctica de la violencia obstétrica en Venezuela.-Los estudios científicos relativos a la situación de la violencia obstétrica en Venezuela son escasos y parciales.

Teniendo a la vista aquella constatación, los informes disponibles evidencian que la violencia obstétrica conserva un elevado grado de prevalencia. En efecto, una investigación desarrollada durante el 2011 y publicada el 2013 concluye que, sobre el universo de pacientes estudiadas en un determinado establecimiento de salud, se constató que el 66,8\% declaró actos médicos sin consentimiento y un $49,4 \%$ declaró trato deshumanizante. Además, solo un 20,5\% reportó atención libre de violencia ${ }^{22}$. Otra investigación, esta vez desarrollada durante el 2012 en un establecimiento de salud diferente y publicada el 2015, indica que el 26,3\% de las mujeres encuestadas sostuvieron haber sido objeto de violencia obstétrica. De ellas declaran "no haber sido tomadas en cuenta ni habérseles informado de manera suficiente en el 65,6\% de las pacientes sobre los procedimientos médicos o quirúrgicos a los cuales fueron sometidas" 23 .

En lo relativo a la información con que cuentan las pacientes, el primero de esos estudios reporta que sólo el 27\% conoce el término violencia obstétrica y solo 19\% sabe dónde denunciarla. Y agrega: “A 6 años de la puesta en marcha de la ley solo un pequeño grupo de mujeres posee la información que le permita empoderarse en su salud sexual y reproductiva. Es necesario difundir los contenidos de la ley para garantizar que se cumplan los fines establecidos en el artículo 2 de la misma, porque si no se hace cumplir la ley, se perpetúa el ciclo de la impunidad"24. La segunda de las mencionadas investigaciones pudo "constatar la dificultad que tienen las mujeres para identificar un trato inadecuado así como tampoco conocen

${ }^{20}$ Artículo 52 de la Ley Orgánica sobre el Derecho de las mujeres a una vida libre de violencia, de Venezuela.

${ }^{21}$ Artículo 52 de la Ley Orgánica sobre el Derecho de las mujeres a una vida libre de violencia, de Venezuela.

22 Terán, Pablo - Castellanos, Carlos - González Blanco, Mireya - RaMos, Damarys, cit. (n. 13), p. 178.

${ }^{23}$ Pereira, Carlota J. - Domínguez, Alexa L. - Toro Merlo, Judith, Violencia obstétrica desde la perspectiva de la paciente, en Revista de Obstetricia y Ginecología de Venezuela 75 (2015), 2, p. 89.

24 Terán, Pablo - Castellanos, Carlos - González Blanco, Mireya - RaMos, Damarys, cit. (n. 13), p. 179. 
$(80,1 . \%)$ cuáles son los mecanismos de denuncia en caso de ser víctimas de violencia obstétrica" 25 . Es probable que aquella dificultad para identificar un trato inadecuado explique en parte el bajo porcentaje de mujeres que declaran haber sido objeto de violencia obstétrica, en comparación con la investigación mencionada en primer lugar.

Por último, conviene destacar que ni siquiera el personal de salud tiene claridad respecto de qué actos son constitutivos de violencia obstétrica. Esto es relevante, porque el mismo personal declara conocer dicho concepto. Esta ignorancia se extiende a los mecanismos de denuncia de estas formas de agresión y las instituciones encargadas de prestar ayuda a las mujeres que la padecen ${ }^{26}$.

\section{Argentina}

a) Regulación de la violencia obstétrica en Argentina.- Argentina cuenta con dos textos legales relacionados con la violencia obstétrica. Cada uno de ellos se complementa con su respectivo reglamento.

El primero de esos textos legales es la ley 25.929, de 2004, conocida también como Ley de Parto Humanizado ${ }^{27}$. Aunque no define qué se entiende por violencia obstétrica, establece una serie de derechos que las mujeres poseen en relación con su embarazo, trabajo de parto, parto y postparto. Entre ellos se mencionan los derechos a ser informada sobre las distintas intervenciones médicas que pudieren tener lugar durante esos procesos de manera que pueda optar libremente cuando existieren diferentes alternativas, a ser tratada con respeto, cautelando su intimidad y considerando sus pautas culturales, a ser considerada una persona sana, al parto natural, a ser informada sobre la evolución de su parto, el estado de su hijo o hija y otros que tienden a asegurar su autonomía jurídica ${ }^{28}$. Esta ley es complementada por un reglamento, que latamente prescribe las acciones que deberá ejecutar el personal de salud a objeto de dar cumplimiento a lo establecido por la Ley de Parto Humanizado ${ }^{29}$.

El segundo de esos textos legales es la ley 26.485, de 2009, ley de pro-

${ }^{25}$ Pereira, Carlota J. - Domínguez, Alexa L. - Toro Merlo, Judith, cit. (n. 23), p. 90.

${ }^{26}$ Faneite, Josmery - Feo, Alejandra - Toro Merlo, Judith, Grado de conocimiento de la violencia obstétrica por el personal de salud, en Revista de Obstetricia y Ginecología de Venezuela 72 (2012), 1, p. 11.

${ }^{27}$ Ley 25.929 (2004), Ley de obligaciones de las obras sociales y medicina prepaga, de Argentina, habitualmente denominada Ley de parto humanizado.

${ }^{28}$ Artículo $2^{\circ}$ de la Ley 25.929 (2004), Ley de obligaciones de las obras sociales y medicina prepaga, de Argentina.

${ }^{29}$ Artículo $2^{\circ}$ del reglamento contenido en el decreto 2035, de Argentina, de 1 de octubre de 2015 . 
tección integral para prevenir, sancionar y erradicar la violencia contra las mujeres en los ámbitos en que desarrollen sus relaciones interpersonales. Al igual que en el caso de Venezuela, este texto normativo define las diferentes modalidades de violencia de género. En cuanto a la violencia obstétrica, la define como "aquélla que ejerce el personal de salud sobre el cuerpo y los procesos reproductivos de las mujeres, expresada en un trato deshumanizado, un abuso de medicalización y patologización de los procesos naturales, de conformidad con la Ley 25.929'30. El reglamento que complementa este texto legal define trato deshumanizado como "el trato cruel, deshonroso, descalificador, humillante o amenazante ejercido por el personal de salud en el contexto de la atención del embarazo, parto y postparto, ya sea a la mujer o allla recién nacidola, asi como en la atención de complicaciones de abortos naturales o provocados, sean punibles o no' ${ }^{\prime 31}$.

En cuanto a las medidas o sanciones frente a la violencia obstétrica, el artículo $6^{\circ}$ de la Ley sobre parto humanizado se limita a prescribir que: "el incumplimiento por parte de los profesionales de la salud y sus colaboradores $y$ de las instituciones en que éstos presten servicios, será considerado falta grave a los fines sancionatorios". Se trata, por tanto, de sanciones administrativas en que la calificación de gravedad es una facultad discrecional de la autoridad que la aplica, esto es, la Dirección de Obras Sociales del Ministerio de Salud. La ley contra la violencia de género, por su parte, no contempla medidas o sanciones pertinentes para situaciones de violencia obstétrica ${ }^{32}$.

Por último, y en el nivel de las políticas públicas, desde el año 2009 se han comenzado a implementar las denominadas Maternidades Seguras y Centradas en la Familia. Se trata de un paradigma organizacional que, en esencia, transforma a los padres y familia en coprotagonistas del proceso de embarazo, parto y puerperio, y estimula el respeto y protección de los derechos de la mujer que se encuentra en dichos contextos ${ }^{33}$.

30 Artículo $6^{\circ}$, letra e), de la Ley 26.485 (2009), de protección integral para prevenir, sancionar y erradicar la violencia contra las mujeres en los ámbitos en que desarrollen sus relaciones interpersonales, de Argentina.

${ }^{31}$ Artículo $6^{\circ}$, letra e), del decreto 1011/2010 reglamentario de la Ley 26.485 sobre protección integral para prevenir, sancionar y erradicar la violencia contra las mujeres, de Argentina.

32 Las sanciones contempladas en el artículo 32 de la Ley 26.485 se dirigen más bien a quien ha incurrido en violencia doméstica. En efecto, dichas sanciones consisten en el llamado de atención al victimario por parte del juez, comunicación del acto cometido al organismo, asociación profesional o lugar de trabajo del agresor y asistencia del mismo a tratamientos terapéuticos tendientes a la modificación de conductas violentas.

33 UNICEF, Maternidad Segura y Centrada en la Familia (MSCF): conceptualización e implementación del modelo (Argentina, 2011), p. 10. 
b) Situación fáctica de la violencia obstétrica en Argentina.- Pese a la regulación y políticas públicas existentes, la violencia obstétrica parece no retroceder de manera significativa en Argentina. Así lo evidencian los indicios proveíos por dos ámbitos de información.

El primero de ellos es la constatada necesidad de generar sanciones más pertinentes y eficaces contra la violencia obstétrica. En efecto, el año 2011 se creó en Argentina la Comisión Nacional Coordinadora de Acciones para la Elaboración de Sanciones de la Violencia de Género (CONSAVIG). Esta comisión tiene por finalidad colaborar en el "diseño de sanciones contra la violencia de género entendiendo que la sanción debe ser una medida reparadora para la(s) víctima(s)". Debido a la especificidad de la violencia obstétrica resultó necesario constituir un grupo de trabajo especial para la materia. De este modo, dos años más tarde, en 2013, se constituyó la Comisión Nacional sobre la Violencia Obstétrica (CONSAVO), dedicada a tratar las sanciones posibles, como reacción ante la aparición de violencia obstétrica ${ }^{34}$.

El segundo ámbito de información son los estudios y estadísticas disponibles, por cierto tan escasos y parciales como en el caso de Venezuela. Un estudio aplicado en una maternidad pública de Buenos Aires el año 2015 permitió concluir que no se habían producido cambios significativos con relación a las investigaciones desarrolladas en 1998 y 2002. Según declara la autora, "fueron relatadas en las entrevistas similares actitudes mecanizadas, impersonales, violentas y contrarias a las MSCF" (Maternidades Seguras y Centradas en la Familia) por parte del equipo de salud, incluso entre los médicos jóvenes ${ }^{35}$. Una de las manifestaciones de esta violencia obstétrica es, según se indicó más arriba, las cesáreas que carecen de justificación sanitaria. Pues bien, en Argentina la tasa de cesáreas sobre el total de partos se encuentra en torno al 25\%, de modo que ocupa el cuarto lugar a nivel Latinoamericano, detrás de Chile, Brasil y República Dominicana ${ }^{36}$. Otro estudio aplicado sobre un grupo de médicos de dos hospitales de Buenos Aires, también el 2015, permite concluir que, en opinión de estos profesionales: "las mujeres de bajo nivel socioeconómico

${ }^{34}$ Galimberti, Diana, Violencia obstétrica (2015), p. 7. Disponible [en línea]: Disponible en: http://www.fasgo.org.ar/images/Violencia_obstetrica.pdf.

35 Morrone, Beatriz, Violencia obstétrica: cuando las Leyes no alcanzan, en II Jornadas de género y diversidad sexual. Ampliación de derechos: proyecciones y nuevos desafios (La Plata, 2016), p. 6; ponencia.

${ }^{36}$ Valenti, Eduardo A. - Laterra, Cristina - Veiga, Angélica - Sosa, Virginia, Análisis de la tasa de cesáreas en una maternidad de tercer nivel a lo largo de 9 años, en Revista de la Federación Argentina de Sociedades de Ginecología y Obstetricia 13 (2014), 1, p. 17. 
y cultural son menos empoderadas e informadas y aceptan lo que ofrecen en el hospital como un favor gratuito". Por su parte: "todos los profesionales, salvo uno, tienen un conocimiento sobre la ley. Sin embargo, sus definiciones de la ley no eran completas" ${ }^{\prime 7}$.

En definitiva, las iniciativas normativas y de políticas públicas tendientes a erradicar la violencia obstétrica y asegurar un parto humanizado no han logrado "fisurar y hacer tambalear una cultura organizacional que violenta los Derechos de las Mujeres, su hijos/as y sus familias", ni "han cambiado las rutinas mecanizadas, a-criticas, medicalizadas y centradas en prácticas instrumentales que van a contrapelo de las Guías internacionales y nacionales"38.

\section{México}

a) Regulación de la violencia obstétrica en México.- En México existen dos órdenes de regulaciones relacionadas con la violencia obstétrica. Una de ellas es de carácter federal y la otra es de nivel estatal.

El Estado federal cuenta, desde el año 2007, con la Ley general de acceso de las mujeres a una vida libre de violencia. Su objetivo es, según declara el artículo $1^{\circ}$ de la misma, prevenir, sancionar y erradicar la violencia contra las mujeres. Aunque este texto normativo carece de una referencia específica a la violencia obstétrica, en sus artículos $6^{\circ}$ y 18 define otras formas de agresión relacionadas con aquella: la violencia psicológica, la violencia física y la violencia que proviene de agentes del Estado y que se denomina violencia institucional. A nivel federal no existe una regulación específica para la violencia obstétrica, aunque existe un proyecto de ley para sancionarla ${ }^{39}$.

A nivel estatal sólo diez de las treinta y un unidades federadas que constituyen el Estado federal han definido la violencia obstétrica en sus respectivas leyes de acceso a una vida libre de violencia. Se trata de los Estados de Chiapas, Veracruz, Chihuahua, Colima, San Luis Potosí, Durango, Guanajuato, Quintana Roo, Tamaulipas e Hidalgo ${ }^{40}$. Como en todos ellos el concepto de violencia obstétrica es similar, a continua-

37 Kaplán Westbrook, Lara, El parto humanizado: perspectivas de profesionales en las maternidades públicas de Buenos Aires, en Independent Study Project (ISP) Collection (2015), p. 39.

${ }^{38}$ Morrone, Beatriz, cit. (n. 35), p. 5.

39 Bellón SÁnchez, Silvia, Obstetric violence: Medicalization, authority abuse and sexism within Spanish obstetric assistance. A new name for old issues? (Tesis de máster, Países Bajos, Utrecht Universiteit, 2014), p. 57.

40 Grupo de Información en Reproducción Elegida (GIRE), Omisión e indiferencia. Derechos reproductivos en México (México D.F., 2013), p. 46. 
ción se reproduce sólo una de esas definiciones: "apropiación del cuerpo y procesos reproductivos de las mujeres por personal de salud, que se expresa en un trato deshumanizador, en un abuso de medicalización y patologización de los procesos naturales, trayendo consigo pérdida de autonomía y capacidad de decidir libremente sobre sus cuerpos y sexualidad"14.

Siempre a nivel estatal, tres de las unidades federadas de México han establecido el delito de violencia obstétrica: Chiapas, Veracruz y Guerrero. Es interesante constatar que las opciones legislativas de cada uno de ellos ha sido diferente. En efecto, el Estado de Chiapas tipifica el delito a partir de su definición y, además, a partir de un listado de conductas que equipara a la violencia obstétrica ${ }^{42}$. El Estado de Veracruz, por su parte, opta por prescribir que comete el delito de violencia obstétrica quien incurre en alguna de las conductas que señala ${ }^{43}$. Por último, el Estado de Guerrero tipifica el delito a partir de una definición de violencia obstétrica ${ }^{44}$. En todos los casos se establecen penas privativas de libertad y multas y, en el caso de Chiapas, se añade la suspensión de la profesión, cargo u oficio, por un término igual, con independencia de las lesiones causadas.

b) Situación fáctica de la violencia obstétrica en México.- Un informe del Grupo GIRE sostiene que no existen estudios o informes cuantitativos de carácter general que permitan dimensionar el problema de la violencia obstétrica en México. Sin embargo, diversas investigaciones académicas con enfoques cualitativos confirman que el tema de la violencia obstétrica se encuentra ausente en las políticas de calidad de los servicios de salud, como así también en la formación de los profesionales de la gineco-obstetricia ${ }^{45}$.

En cuanto a la ausencia de políticas de calidad de los servicios de salud, un estudio que analizó la percepción de mujeres atendidas en el año 2012 en dos hospitales del Estado de Morelos permitió concluir que el 19,34\% sostuvo haber sido víctima de violencia verbal, un 7,61\% de violencia física y un $29,10 \%$ de violencia total. Evaluando otra manifestación de violencia obstétrica, esta vez de carácter sicológico, el mismo estudio reveló que el $98 \%$ de las mujeres declaró que habían sido objeto de 5 tactos vaginales en promedio, con un mínimo de uno y un máximo de 20 de tales pro-

${ }^{41}$ Artículo 6 de la Ley de acceso a una vida libre de violencia para las mujeres en el Estado de Chiapas México.

${ }^{42}$ Artículos 183 ter y 183 quater del Código Penal de Chiapas.

43 Artículo 363 del Código Penal de Veracruz.

${ }^{44}$ Artículo 202 del Código Penal de Guerrero.

${ }^{45}$ Grupo de Información en Reproducción Elegida (GIRE), cit. (n. 40), p. 126. 
cedimientos durante su proceso perinatal ${ }^{46}$. Adviértase la elevada cifra de esta intensa e injustificada forma de invasión en la intimidad de la mujer.

En cuanto a la formación de los profesionales de la gíneco-obstetricia, Castro destaca que el currículum oculto de los estudios de medicina en México viabiliza que los médicos incurran en violencia obstétrica. $\mathrm{Al}$ respecto concluye su investigación sociológica preguntándose: "qué posibilidades tienen los prestadores de servicios de salud de establecer relaciones ciudadanas con las usuarias de los servicios, es decir, relaciones basadas en la igualdad, en el conocimiento y el reconocimiento de los diversos derechos de salud y reproductivos, si ellos han sido incorporados plenamente en un campo autoritario y jerárquico y, por lo tanto, han incorporado ellos mismos la lógica de dicho campo" "47. Aquella formación jerárquica y autoritaria es la que, probablemente, genera resistencias entre los médicos mexicanos hacia los procedimientos de parto humanizado que se han intentado incorporar en el país ${ }^{48}$.

\section{Chile}

a) Regulación de la violencia obstétrica en Chile.- Chile carece de una legislación que enfrente de manera directa el grave fenómeno de la violencia obstétrica. Sin perjuicio de aquella afirmación, resulta conveniente revisar ciertas normas de la Ley 20.584 y de sus reglamentos que parecen presentar vinculación con dicho problema. Adicionalmente, y para una mejor ilustración, conviene revisar el único proyecto de ley en actual tramitación parlamentaria que pretende hacer frente a esta forma de agresión contra la mujer. Por último, se hará referencia a la lex artis médica y su incidencia en la violencia obstétrica.

Primero; la Ley 20.584, que regula los derechos y deberes que tienen las personas en relación con acciones vinculadas a su atención en salud, contiene tres órdenes de regulación de interés para la presente investigación.

De un lado, establece un conjunto de derechos a favor de los pacientes. Ninguno de ellos se refiere de manera expresa a la protección de la mujer

46 Valdez-Santiago, Rosario - Hidalgo-Solórzano, Elisa - Mojarro-IÑIguez, Mariana - Arenas-Monreal, Luz María, Nueva evidencia a un viejo problema: el abuso de las mujeres en las salas de parto, en Revista CONAMED 18 (2013), 1, pp. 18-19.

${ }^{47} \mathrm{Y}$ agrega el autor: "Este enfoque, desde luego, no exime de responsabilidad a los actores (los médicos y enfermeras en particular) de los atropellos en que incurren, pero sí nos permite comprender científicamente el origen social de los resortes que impulsan sus actuaciones"; CASTro, Roberto, Génesis y práctica del habitus médico autoritario en México, en Revista Mexicana de Sociología 76 (2014), 2, p. 191.

48 Pozzio, María Raquel, La gíneco-obstetricia en México: entre el "parto humanizado" y la violencia obstétrica, en Revista Estudios Feministas 24 (2016), 1, p. 107. 
en situación perinatal frente a la violencia obstétrica. Su intención fue, más bien y en primer lugar, el respeto a los pacientes y el resguardo de su privacidad $^{49}$. Y, en segundo lugar, el reconocimiento de la autonomía de las personas, promoviendo su participación activa en su proceso de atención en salud ${ }^{50}$. Con todo, no está claro si la posibilidad de rechazar un tratamiento $^{51}$, lo que se extiende a todo procedimiento sanitario ${ }^{52}$, incluye el derecho a elegir la clase de parto por la que se opta (natural o cesárea), ni la posición en que se desea enfrentar dicho proceso.

De otro lado, la Ley 20.584 incluye un procedimiento de reclamo administrativo. En particular confiere el derecho a reclamo respecto de la atención de salud recibida, sienta las bases del procedimiento para hacerlo efectivo $^{53}$, y delega en un reglamento la regulación de este último ${ }^{54}$. Ni la ley

49 Valenzuela, Sergio - Aliaga, Verónica - Burdiles, Patricio - Carvallo, Aurelio - Díaz, Eduardo - Guerrero, Manuel - Rueda, Laura - Valenzuela, Carlos, Reflexiones en torno a la Ley 20.584 y sus implicancias para la investigación biomédica en Chile, en Revista Médica de Chile 143 (2015), 1, p. 96. En este sentido la legislación se limita a disponer que las personas tienen derecho a recibir un trato digno y respetuoso en todo momento y en cualquier circunstancia (Ley 20.584, artículo $5^{\circ}$, inciso primero e inciso segundo letra b), y decreto 38, de 17 de julio de 2012, que aprueba reglamento sobre derechos y deberes de las personas en relación a las actividades vinculadas con su atención de salud, artículo $5^{\circ}$ ), y prescribe que los prestadores de salud deberán asegurar el respeto y protección de la vida privada y la honra de la persona durante su atención de salud (Ley 20.584, artículo $5^{\circ}$, inciso segundo, letra c) y decreto 38, de 17 de julio de 2012, que aprueba reglamento sobre derechos y deberes de las personas en relación a las actividades vinculadas con su atención de salud, artículo $7^{\circ}$ ). La Ley confiere también el derecho a que el paciente esté acompañado de familiares y amigos cercanos durante su hospitalización (Ley 20.584, artículo $6^{\circ}$ ), lo que el respectivo reglamento rebaja al calificarlo de derecho a ser "visitado" (decreto 38, de 17 de julio de 2012, que aprueba reglamento sobre derechos y deberes de las personas en relación a las actividades vinculadas con su atención de salud, artículo 10).

${ }^{50}$ Valenzuela, Sergio - Aliaga, Verónica - Burdiles, Patricio - Carvallo, Aurelio - Díaz, Eduardo - Guerrero, Manuel - Rueda, Laura - Valenzuela, Carlos, cit. (n. 49), p. 96. Al respecto la Ley otorga el derecho a ser informado de las alternativas de tratamiento disponibles para su recuperación y de los riesgos que ello pueda representar (Ley 20.584, artículo 10, decreto 38, de 17 de julio de 2012, que aprueba reglamento sobre derechos y deberes de las personas en relación a las actividades vinculadas con su atención de salud, artículo 12, y decreto 31, de 15 de junio de 2012, que aprueba reglamento sobre entrega de información y entrega de consentimiento informado en las atenciones de salud, artículo $1^{\circ}$, inciso primero).

51 Ley 20.584, artículo 14.

52 LeÓN C., Francisco Javier, Ley de derechos y deberes de las personas en la atención de salud. Una mirada bioética, en Revista Médica de Chile 140 (2012), 11, p. 1492.

${ }^{53}$ Ley 20.584, artículo 37.

${ }^{54}$ Ley 20.584, artículo 30, incisos primero y segundo. 
ni sus reglamentos aluden a medidas de reparación a favor de los pacientes, aunque le otorgan la posibilidad de recurrir ante la Superintendencia de Salud en caso de no resultar satisfechas sus pretensiones ante el prestador ${ }^{55}$.

Por último, la legislación se preocupa de regular los posibles casos de maltrato o violencia. Sin embargo, lo hace desde los pacientes hacia el personal de salud y no a la inversa. Al respecto dispone que se pueden ejercer las acciones civiles y penales que correspondan en contra de quienes incurran en trato irrespetuoso o en actos de violencia verbal o física respecto de los integrantes del equipo de salud, e incluso se puede solicitar el auxilio de la fuerza pública ${ }^{56}$. Sin aludir a posibles formas de violencia en contra de los pacientes, la ley dispone que los prestadores institucionales públicos deberán hacer efectiva la responsabilidad administrativa de sus funcionarios que no respeten los derechos de los pacientes ${ }^{57}$. Sin embargo, no incluye normas para el caso que esta vulneración se produzca en el sector privado de salud.

Segundo; junto con esas normas que son ley de la República, resulta conveniente hacer una concisa referencia al proyecto de ley en actual tramitación parlamentaria relativo a la violencia obstétrica ${ }^{58}$.

$\mathrm{Al}$ respecto se debe destacar que se trata de una iniciativa que "busca consagrar los derechos de las mujeres en relación con el trabajo de parto, el parto y el postparto" 59 . En dicho proyecto se define la violencia ginecoobstétrica $^{60}$, se señalan por vía ejemplar las conductas que la constituyen ${ }^{61}$, se establecen derechos a favor de la mujer en situación perinatal, del padre

55 Ley 20.584, artículo 37, inciso segundo, y decreto 35, de 4 de julio de 2012, que aprueba reglamento sobre el procedimiento de reclamo de la Ley 20.584, que regula los derechos y deberes que tienen las personas en relación con acciones vinculadas a su atención en salud, artículo 14.

${ }^{56}$ Ley 20.584, artículo 35, inciso tercero y decreto 38, de 17 de julio de 2012, que aprueba reglamento sobre derechos y deberes de las personas en relación a las actividades vinculadas con su atención de salud, artículo 25 .

57 Ley 20.584, artículo 38, inciso primero y decreto 38, de 17 de julio de 2012, que aprueba reglamento sobre derechos y deberes de las personas en relación a las actividades vinculadas con su atención de salud, artículo 13.

58 Proyecto de Ley iniciado por moción parlamentaria presentado ante la Cámara de Diputados el 28 de enero de 2015, Boletín $N^{\circ}$ 9902-11. En su artículo primero regula lo que denomina violencia gineco-obstétrica, mientras que en el artículo segundo introduce una modificación al Código Penal para sancionar el delito de violencia obstétrica.

59 Instituto Nacional de Derechos Humanos, Situación de los derechos humanos en Chile (Informe anual, 2016), p. 239.

${ }^{60}$ Artículos $4^{\circ}$ y $6^{\circ}$ del proyecto de Ley boletín $N^{\circ} 9902-11$.

${ }^{61}$ Artículo $5^{\circ}$ del proyecto de Ley boletín $\mathrm{N}^{\circ}$ 9902-11. 
y de otros familiares cercanos ${ }^{62}$, se impone la obligación de informar sobre los deberes del personal de salud y los derechos de las pacientes ${ }^{63}$, se establecen las sanciones de carácter penal que proceden contra el personal de salud $^{64} \mathrm{y}$ se fijan normas procesales para hacer efectiva la responsabilidad civil y penal derivadas de esta forma de violencia de género ${ }^{65}$.

Pese a la relevancia de esta iniciativa, desde la fecha de su presentación (enero de 2015) no ha tenido avance alguno ${ }^{66}$.

Tercero; la lex artis médica puede constituir un relevante referente al momento de enfrentar el problema de la violencia obstétrica. Sin embargo, se limita al "conjunto de normas o criterios valorativos que el medico en posesión de conocimientos, habilidades y destrezas debe aplicarlos diligentemente en la situación concreta de un enfermo y que han sido universalmente aceptadas por sus pares" ${ }^{67}$. Por lo mismo, y considerando de un lado que la violencia obstétrica proviene del personal sanitario en general y no sólo de los médicos y, de otro lado, que de lo que se trata es de empoderar a las mujeres en contextos perinatales y a sus familias, la lex artis aparece evidentemente insuficiente para enfrentar este polisémico problema.

b) Situación fáctica de la violencia obstétrica en Chile.- Los estudios realizados por Michelle Sadler, probablemente la principal investigadora de Chile sobre la violencia obstétrica, permiten concluir que se trata de una situación extendida en el país. Sostiene que aquella es el resultado institucional de paradigma tecnocrático de atención que domina en hospitales públicos y en clínicas privadas. Este paradigma, precisa, expresa los valores centrales de las sociedades occidentales: "una fuerte orientación hacia la ciencia, alta tecnología, beneficio económico, e instituciones gobernadas por un poder patriarcal" 68 . Las prácticas de violencia obstétrica en el país no son únicamente resultado de conductas individuales del personal de salud, sino de todo el proceso de enseñanza universitaria y de práctica profesional de esos mismos profesionales. Es el "currículum oculto", que

\footnotetext{
${ }^{62}$ Artículos $2^{\circ}, 7^{\circ}$ y $8^{\circ}$ del proyecto de Ley boletín $N^{\circ} 9902-11$.

${ }^{63}$ Artículos $9^{\circ}$ y 12 del proyecto de Ley boletín $\mathrm{N}^{\circ}$ 9902-11.

${ }^{64}$ Artículos 10 y 11 del proyecto de Ley boletín N 9902-11.

${ }^{65}$ Artículos 13 y 14 del proyecto de Ley boletín $N^{\circ} 9902-11$.

${ }^{66}$ El proyecto pasó a la Comisión de Salud en marzo de 2015 para informe. Luego de ello no ha tenido avances.

${ }_{67}$ Colegio Médico de Chile, Consideraciones sobre lex artis. Error y negligencias médicas, p. 1. Disponible [en línea]: http://www.colegiomedico.cl/wp-content/ uploads/2015/08/081112lex_artis_medica.doc.

${ }^{68}$ SAdler Spencer, Michelle, Etnografías del control del nacimiento en el Chile contemporáneo, en Revista Chilena de Antroplogía 33 (2016), 1, p. 47.
} 
ya fuera mencionado en el caso de México, uno de los factores esenciales en la actual situación en Chile ${ }^{69}$.

Tal vez el principal dato estadístico relevante sobre la materia, por lo demás manifestación de violencia obstétrica, es la cantidad de cesáreas que se aplican en el país. De hecho los propios médicos reconocen que se ha producido un "alarmante aumento en el número de cesáreas" en los últimos años ${ }^{70}$, que el Instituto Nacional de Derechos Humanos ha cifrado para el año 2015 en un 40,9\% del total de partos atendidos en el sector público y en un 69,0\% del total de partos atendidos en el sector privado $^{71}$. Al respecto se debe recordar que, según se indicó más arriba, la Organización Mundial de la Salud ha recomendado que las cesáreas no excedan del 15\% del total de partos.

\section{CONCLUSIONES}

La pregunta que ha dado inicio a la presente investigación fue formulada de la siguiente manera en la introducción de este trabajo: Cuál es la situación normativa y fáctica de la violencia obstétrica en los países de América Latina que han legislado sobre la materia, y particularmente en Chile?

En respuesta a esa interrogante, los datos obtenidos durante el desarrollo de la investigación permiten arribar a las siguientes conclusiones:

a) La gran mayoría de los países de América Latina carecen de una regulación que haga frente de manera directa a la violencia obstétrica.

b) Sólo tres países de dicho entorno han aprobado leyes directamente relacionadas con la violencia obstétrica: Venezuela, Argentina y México. En el caso de este último no se trata de legislaciones de nivel federal, sino a nivel de sus unidades federadas.

c) Aunque los estudios empíricos disponibles son, según se ha dicho, escasos y parciales, entregan coincidentes indicios respecto de la situación fáctica de la violencia obstétrica en los tres países revisados. A partir de tales indicios no resulta posible dimensionar la extensión, gravedad ni enraizamieto de esta forma de violencia. Sin embargo, a partir de los mismos parece posible sostener que las diversas conductas constitutivas

69 Sadler Spencer, Michelle, cit. (n. 68), p. 53; y Sadler, Michelle - Santos, Mário J.D.S. - Ruiz-Berdún, Dolores - Leiva Rojas, Gonzalo - Sкоко, Elena Gillén, Patricia - Clausen, Jette A., cit. (n. 4), p. 49., p. 51.

${ }^{70}$ Canessa B., Eugenio - Añazco G., Rodrigo - Gigoux M., Jorge - Aguilera S., Jorge, Anestesia para el trabajo de parto, en Revista Médica Clínica Las Condes 25 (2014), 6, p. 984.

${ }^{71}$ Instituto Nacional de Derechos Humanos, cit. (n. 50), pp. 239 y 244247, tablas 2 y 3 . 
de violencia obstétrica subsisten, pese a la incorporación de las mencionadas legislaciones. Los mismos estudios revelan la prevalencia de culturas institucionales y profesionales contranormativas en los establecimientos de salud investigados.

d) Chile carece de una legislación específica para enfrentar la violencia obstétrica. La ley 20.584, de 2012, sobre derechos y deberes de los pacientes, recoge algunos derechos aplicables al ámbito perinatal. Sin embargo, la única forma de violencia que expresamente reconoce y regula es aquella en que puede incurrir un paciente en contra del personal de salud, pero no a la inversa. El proyecto de ley que pretende proteger a la mujer en situación de embarazo, parto y postparto fue presentado el 2015 y carece de tramitación desde entonces.

e) A partir de lo sostenido en la presente conclusión, resulta posible sostener al menos dos cosas en el caso de Chile. En primer lugar, que la experiencia comparada indica que la violencia obstétrica no puede ser resuelta mediante normas, pues se requiere un cambio cultural. Esto significa difusión de derechos en la ciudadanía, adecuaciones en los planes de estudio de quienes se desempeñan en el ámbito de la salud (modificación del currículum oculto) y la institucionalización de procedimientos sanitarios que impidan la victimización de la mujer en contextos perinatales, entre otras medidas. En segundo lugar, que ni la legislación vigente, ni el proyecto de ley sobre violencia obstétrica en actual tramitación parlamentaria se hacen cargo de esos aspectos.

\section{BibLIOGRAFÍA}

Arguedas Ramírez, Gabriela, La violencia obstétrica: propuesta conceptual a partir de la experiencia costarricense, en Cuadernos Inter.c.a.mbio sobre Centroamérica y el Caribe 11 (2014), 1.

BELLI, Laura, La violencia obstétrica: otra forma de violación a los derechos humanos, en Redbioética 4 (2013), 1.

Bellón SÁnChez, Silvia, Obstetric violence: Medicalization, authority abuse and sexism within Spanish obstetric assistance. A new name for old issues? (Tesis de máster, Países Bajos, Utrecht Universiteit, 2014).

Benítez Guerra, Gidder, Violencia obstétrica, en Revista de la Facultad de Medicina de la Univesidad Central de Venezuela 31 (2008), 1.

Camacaro, M., Patologizando lo natural, naturalizando lo patológico... improntas de la praxis obstétrica, en Revista venezolana de estudios de la mujer 14 (2009), 32.

Canessa B., Eugenio - Añazco G., Rodrigo - Gigoux M., Jorge - Aguilera S., Jorge, Anestesia para el trabajo de parto, en Revista Médica Clínica Las Condes 25 (2014), 6.

Castro, Roberto, Génesis y práctica del habitus médico autoritario en México, en Revista Mexicana de Sociología 76 (2014), 2.

Colegio Médico de Chile, Consideraciones sobre lex artis. Error y negligencias 
médicas. Disponible [en línea]: http://www.colegiomedico.cl/wp-content/ uploads/2015/08/081112lex_artis_medica.doc.

Faneite, Josmery - Feo, Alejandra - Toro Merlo, Judith, Grado de conocimiento de la violencia obstétrica por el personal de salud, en Revista de Obstetricia y Ginecología de Venezuela 72 (2012), 1.

FernÁNDez Guillén, Francisca, Nosotras parimos, ¿nosotras decidimos? El consentimiento de la mujer y otros aspectos legales de la atención materno infantil, en Medicina Naturista 10 (2006).

GalimberTi, Diana, Violencia obstétrica (2015). Disponible [en línea]: http://www. fasgo.org.ar/images/Violencia_obstetrica.pdf.

Grupo de Información en Reproducción Elegida (GIRE), Omisión e indiferencia. Derechos reproductivos en México (México D.F., 2013). Disponible [en línea]: http://www.sitioswwweb.com/miguel/Gire-Aborto.pdf.

KaPLÁN WeSTBROOK, Lara, El parto humanizado: perspectivas de profesionales en las maternidades públicas de Buenos Aires, en Independent Study Project (ISP) Collection (2015). Disponible [en línea]: http://digitalcollections.sit.edu/cgi/viewcontent. cgi?article=3272\& context=isp_collection.

León C., Francisco Javier, Ley de derechos y deberes de las personas en la atención de salud. Una mirada bioética, en Revista Médica de Chile 140 (2012), 11.

Medina, Graciela, Violencia obstétrica (2009). Disponible [en línea]: http://www. gracielamedina.com/violencia-obst-trica/.

Morrone, Beatriz, Violencia obstétrica: cuando las leyes no alcanzan, en Jornadas de género y diversidad sexual. Ampliación de derechos: proyecciones y nuevos desafios (La Plata, 2016), Disponible [en línea]: http://www.trabajosocial.unlp.edu.ar/uploads/ docs/violenca_obstetrica_cuando_las_leyes_no_alcanzan_.pdf.

Observatorio de Equidad de Género en Salud. Violencia de Género en Chile, Informe monográfico 2007-2012 (4º versión, Santiago, OPS/OMS, 2013). Disponible [en línea]: http://www.paho.org/chi./index.php?option=com_docman\&task=doc_ view\&gid=123\&Itemid.

Organización Mundial de la Salud, Declaración de la OMS sobre tasas de cesárea (1985). Disponible [en línea]: http://apps.who.int/iris/bitstream/10665/161444/1/ WHO_RHR_15.02_spa.pdf.

Pereira, Carlota J. - Domínguez, Alexa L. - Toro Merlo, Judith, Violencia obstétrica desde la perspectiva de la paciente, en Revista de Obstetricia y Ginecología de Venezuela 75 (2015), 2.

Pozzio, María Raquel, La gineco-obstetricia en México: entre el "parto humanizado" y la violencia obstétrica, en Revista Estudios Feministas 24 (2016), 1.

SAder Spencer, Michelle, Etnografias del control del nacimiento en el Chile contemporáneo, en Revista Chilena de Antroplogía 33 (2016), 1.

Sadler, Michelle - Santos, Mário J.D.S. - Ruiz-Berdún, Dolores - Leiva Rojas, Gonzalo - Sкоко, Elena - Gillén, Patricia - Clausen, Jette A., Moving beyond disrespect and abuse: addressign the structural dimension of obstetric violence, en Reproductive Health Matters 24 (2016).

Terán, Pablo - Castellanos, Carlos - González Blanco, Mireya - Ramos, Damarys, Violencia obstétrica: percepción de las usuarias, en Revista de Obstetricia y Ginecología de Venezuela 73 (2013), 3.

UNICEF, Maternidad Segura y Centrada en la Familia (MSCF): conceptualización e 
implementación del modelo (Argentina, 2011). Disponible [en línea]: https://www. unicef.org/argentina/spanish/GUIA_MSCF.pdf.

Valdez-Santiago, Rosario - Hidalgo-Solórzano, Elisa - Mojarro-Iñiguez, Mariana, - Arenas-Monreal, Luz María, Nueva evidencia a un viejo problema: el abuso de las mujeres en las salas de parto, en Revista CONAMED 18 (2013), 1.

Valenti, Eduardo A. - Laterra, Cristina - Veiga, Angélica - Sosa, Virginia, Análisis de la tasa de cesáreas en una maternidad de tercer nivel a lo largo de 9 años, en Revista de la Federación Argentina de Sociedades de Ginecología y Obstetricia 13 (2014), 1. Valenzuela, Sergio - Aliaga, Verónica - Burdiles, Patricio - Carvallo, Aurelio - Díaz, Eduardo - Guerrero, Manuel - Rueda, Laura - Valenzuela, Carlos, Reflexiones en torno a la ley 20.584 y sus implicancias para la investigación biomédica en Chile, en Revista Médica de Chile 143 (2015), 1. 
